\title{
DIRAC EQUATION IN SPACETIMES WITH NON-METRICITY AND TORSION
}

\author{
M. Adak \\ Department of Physics, Pamukkale University, \\ 20100 Denizli, Turkey \\ madak@pamukkale.edu.tr \\ T. Dereli \\ Department of Physics, Koç University, \\ 80910 Sarıyer, İstanbul, Turkey \\ tdereli@ku.edu.tr \\ L. H. Ryder \\ Department of Physics, University of Kent, \\ Canterbury, Kent CT2 7NF, UK \\ l.h.ryder@ukc.ac.uk
}

October 25, 2018

\begin{abstract}
Dirac equation is written in a non-Riemannian spacetime with torsion and non-metricity by lifting the connection from the tangent bundle to the spinor bundle over spacetime. Foldy-Wouthuysen transformation of the Dirac equation in a Schwarzschild background spacetime is considered and it is shown that both the torsion and non-metricity couples to the momentum and spin of a massive, spinning particle. However, the effects are small to be observationally significant.
\end{abstract}




\section{Introduction}

Einstein's general relativity provides an elegant (pseudo-)Riemannian formulation of gravitation in the absence of matter. In the variational approach, Einstein's field equations are obtained by considering variations of the Einstein-Hilbert action with respect to the metric and its associated Levi-Civita connection of the spacetime. That is, the absence of matter means that the connection is metric compatible and torsion free, a situation which is natural but not always convenient. A number of developments in physics in recent years suggest the possibility that the treatment of spacetime might involve more than a Riemannian structure:

- The vain effort to quantize gravity with standard field theoretic methods. This is perhaps so far the strongest piece of evidence for going beyond a geometry dominated by classical metric concepts.

- The generalization of the theory of elastic continua to 4-dimensional spacetime. This may provide a physical interpretation of the non-Riemannian structures which emerge in the theory.

- The study of the early universe, in particular singularity theorems, gave us clues for solving the problem of unification of all interactions and the related problem of compactification of dimensions. Inflationary models with dilaton-induced Weyl co-vector is another problem where we meet nonRiemannian structures.

- The description of hadronic (or nuclear) matter in terms of extended structures. The string theories are the best examples.

In the framework of string theories there are hints that by using non-Riemannian geometry we may accommodate several degrees of freedom coming from the low energy limit of string interactions in terms of a connection with non-metricity and torsion [1]. It is interesting to observe that, since string theories are expected to produce effects which are at least in principle testable at low energies, there may be chances to obtain non-Riemannian models with predictions that can be tested.

Although theories in which the non-Riemannian geometrical fields are dynamical in the absence of matter are more elusive to interpret, they may play an important role in certain astrophysical contexts. Part of the difficulty in interpreting such fields is that there is little experimental guidance available for the construction of a viable theory that can compete effectively with general relativity in domains that are currently accessible to observation. In such circumstances one must be guided by the classical solutions admitted by theoretical models that admit dynamical non-Riemannian structures [2, 3, 国].

In the next section we give some basic concepts in differential geometry and mathematical tools in order to perform the calculations given later in the paper. In section 3 we write down the Dirac equation in non-Riemannian spacetimes with non-vanishing curvature, torsion and non-metricity by the Kosmann lift of the connection given in the frame bundle to the spinor bundle [5, 6, [7]. The physical 
meaning of curvature is well-known. In order to gain insight concerning torsion and non-metricity we investigate the low energy limit of the Dirac equation [8]. While doing this we perform the Foldy-Wouthuysen transformation [9, 10, 11]; thus uncoupling the positive and the negative energy states of the Dirac equation. The final section is reserved for discussion.

\section{Non-Riemannian spacetime}

Spacetime is denoted by the triple $\{M, g, \nabla\}$ where $\mathrm{M}$ is a 4-dimensional differentiable manifold, equipped with a Lorentzian metric $g$ which is a second rank, covariant, symmetric, non-degenerate tensor and $\nabla$ is a linear connection which defines parallel transport of vectors (or more generally tensors). With an orthonormal basis $\left\{X_{a}\right\}$,

$$
g=\eta_{a b} e^{a} \otimes e^{b} \quad, \quad a, b, \cdots=0,1,2,3
$$

where $\eta_{a b}$ is the Minkowski metric which is a matrix whose diagonal terms are $-1,1,1,1$ and $\left\{e^{a}\right\}$ is the orthonormal co-frame. The local orthonormal frame $\left\{X_{a}\right\}$ is dual to the co-frame $\left\{e^{a}\right\}$;

$$
e^{b}\left(X_{a}\right)=\delta_{a}^{b}
$$

The spacetime orientation is set by the choice $\epsilon_{0123}=+1$. In addition, the connection is specified by a set of connection 1 -forms $\Lambda_{b}^{a}$. The non-metricity 1-forms, torsion 2-forms and curvature 2-forms are defined through the Cartan structure equations

$$
\begin{aligned}
2 Q_{a b} & :=-D \eta_{a b}=\Lambda_{a b}+\Lambda_{b a}, \\
T^{a} & :=D e^{a}=d e^{a}+\Lambda^{a}{ }_{b} \wedge e^{b}, \\
R_{b}^{a} & :=D \Lambda_{b}^{a}:=d \Lambda_{b}^{a}{ }_{b}+\Lambda^{a}{ }_{c} \wedge \Lambda_{b}^{c} .
\end{aligned}
$$

$\mathrm{d}, \mathrm{D}, \imath_{a}, *$ denote the exterior derivative, the covariant exterior derivative, the interior derivative and the Hodge star operator, respectively. The linear connection 1 -forms can be decomposed uniquely as follows [4]

$$
\Lambda_{b}^{a}=\omega_{b}^{a}+K_{b}^{a}+q_{b}^{a}+Q_{b}^{a}
$$

where $\omega_{b}^{a}$ are the Levi-Civita connection 1-forms that satisfy

$$
d e^{a}+\omega^{a}{ }_{b} \wedge e^{b}=0,
$$

$K_{b}^{a}$ are the contortion 1-forms such that

$$
K^{a}{ }_{b} \wedge e^{b}=T^{a},
$$

and $q_{b}^{a}$ are the anti-symmetric tensor 1 -forms defined by

$$
q_{a b}=-\left(\imath_{a} Q_{b c}\right) \wedge e^{c}+\left(\imath_{b} Q_{a c}\right) \wedge e^{c} .
$$


In the above decomposition the symmetric part

$$
\Lambda_{(a b)}=Q_{a b}
$$

while the anti-symmetric part

$$
\Lambda_{[a b]}=\omega_{a b}+K_{a b}+q_{a b} .
$$

It is cumbersome to take into account all components of non-metricity and torsion in gravitational models. Therefore we will be content with dealing only with certain irreducible parts of them to gain physical insight. The irreducible decompositions of torsion and non-metricity invariant under the Lorentz group are summarily given below. For details one may consult Ref. [3]. The nonmetricity 1-forms $Q_{a b}$ can be split into their trace-free $\bar{Q}_{a b}$ and the trace parts as

$$
Q_{a b}=\bar{Q}_{a b}+\eta_{a b} Q
$$

where the Weyl 1-form $Q=Q^{a}{ }_{a}$ and $\eta^{a b} \bar{Q}_{a b}=0$. Let us define

$$
\begin{aligned}
& \Lambda_{b}:=\imath_{a} \bar{Q}_{b}^{a}, \Lambda:=\Lambda_{a} e^{a}, \\
& \Theta_{b}:={ }^{*}\left(\bar{Q}_{a b} \wedge e^{a}\right), \quad \Theta:=e^{b} \wedge \Theta_{b}, \quad \Omega_{a}:=\Theta_{a}-\frac{1}{3} \imath_{a} \Theta
\end{aligned}
$$

as to use them in the decomposition of $Q_{a b}$ as

$$
Q_{a b}=Q_{a b}^{(1)}+Q_{a b}^{(2)}+Q_{a b}^{(3)}+Q_{a b}^{(4)}
$$

where

$$
\begin{aligned}
Q_{a b}^{(2)} & =\frac{1}{3}{ }^{*}\left(e_{a} \wedge \Omega_{b}+e_{b} \wedge \Omega_{a}\right) \\
Q_{a b}^{(3)} & =\frac{2}{9}\left(\Lambda_{a} e_{b}+\Lambda_{b} e_{a}-\frac{1}{2} \eta_{a b} \Lambda\right) \\
Q_{a b}^{(4)} & =\frac{1}{4} \eta_{a b} Q \\
Q_{a b}^{(1)} & =Q_{a b}-Q_{a b}^{(2)}-Q_{a b}^{(3)}-Q_{a b}^{(4)} .
\end{aligned}
$$

We have $\imath^{a} Q_{a b}^{(1)}=\imath^{a} Q_{a b}^{(2)}=0, \quad \eta^{a b} Q_{a b}^{(1)}=\eta^{a b} Q_{a b}^{(2)}=\eta^{a b} Q_{a b}^{(3)}=0, \quad$ and $e^{a} \wedge Q_{a b}^{(1)}=0$. In a similar way the irreducible decomposition of $T^{a}$ 's invariant under the Lorentz group are given in terms of

$$
\alpha=\imath_{a} T^{a}, \quad \sigma=e_{a} \wedge T^{a}
$$

so that

$$
T^{a}=T^{a(1)}+T^{a(2)}+T^{a(3)}
$$


where

$$
\begin{aligned}
T^{a(2)} & =\frac{1}{3} e^{a} \wedge \alpha, \\
T^{a(3)} & =\frac{1}{3} \imath^{a} \sigma, \\
t^{a} & :=T^{a(1)}=T^{a}-T^{a(2)}-T^{a(3)} .
\end{aligned}
$$

Here $\imath_{a} t^{a}=\imath_{a} T^{a(3)}=0, \quad e_{a} \wedge t^{a}=e_{a} \wedge T^{a(2)}=0$. To give the contortion components in terms of the irreducible components of torsion, we firstly write

$$
2 K_{a b}=\imath_{a} T_{b}-\imath_{b} T_{a}-\left(\imath_{a} \imath_{b} T_{c}\right) e^{c}
$$

from (\$) and then substituting (20) into above we find

$$
\begin{aligned}
2 K_{a b}= & \imath_{a} t_{b}-\imath_{b} t_{a}-\left(\imath_{a} \imath_{b} t_{c}\right) e^{c} \\
& +\frac{2}{3}\left(e_{a} \wedge \imath_{b} \alpha-e_{b} \wedge \imath_{a} \alpha\right) \\
& +\frac{2}{3}\left(\imath_{a} \imath_{b} \sigma\right)-\frac{1}{3}\left(\imath_{a} \imath_{b} \imath_{c} \sigma\right) e^{c} .
\end{aligned}
$$

In components $K_{a b}=K_{c, a b} e^{c}, \quad t_{a}=\frac{1}{2} t_{b c, a} e^{b c}, \quad \alpha=F_{a} e^{a}, \quad \sigma=\frac{1}{3 !} \sigma_{a b c} e^{a b c}$ this becomes

$$
\begin{aligned}
K_{c, a b}= & \frac{1}{2}\left(t_{a b, c}-t_{b c, a}+t_{a c, b}\right) \\
& +\frac{1}{3}\left(F_{b} \eta_{a c}-F_{a} \eta_{b c}\right)-\frac{1}{6} \sigma_{a b c} .
\end{aligned}
$$

\section{Dirac equation}

Since gauge theories seem important for the description of fundamental interactions it appears natural to exploit any gauge structure present in theories of gravity. Different authors, however, adopt different criteria in order to determine what properties a theory should possess in order for it to qualify as a gauge theory. We take the gravitational gauge group to be the local Lorentz group [2] and do calculations in terms of $\mathbf{S p i n}_{+}(3,1)$ structure group, that is the double covering of Lorentz group, keeping in mind the inclusion of spin structure. We will work with exterior forms taking their values in the $\mathcal{C} \ell_{3,1}$ Clifford algebra. $\mathcal{C} \ell_{3,1}$ with an orthonormal basis $\left\{\gamma_{0}, \gamma_{1}, \gamma_{2}, \gamma_{3}\right\}$ is generated by the relations

$$
\gamma^{a} \gamma^{b}+\gamma^{b} \gamma^{a}=2 \eta^{a b} I_{4 \times 4}
$$

where we use the following Dirac matrices

$$
\begin{aligned}
& \gamma_{0}=i\left(\begin{array}{cc}
-I & 0 \\
0 & I
\end{array}\right), \quad \gamma_{1}=i\left(\begin{array}{cc}
0 & \sigma^{1} \\
-\sigma^{1} & 0
\end{array}\right) \\
& \gamma_{2}=i\left(\begin{array}{cc}
0 & \sigma^{2} \\
-\sigma^{2} & 0
\end{array}\right), \quad \gamma_{3}=i\left(\begin{array}{cc}
0 & \sigma^{3} \\
-\sigma^{3} & 0
\end{array}\right) .
\end{aligned}
$$


Here $\sigma^{i}$ are the Pauli matrices.

A 4-component Dirac spinor is a section of the spinor bundle over spacetime whose fibers are $\mathbb{C}^{4}$. This bundle is simply the vector bundle associated to the tangent bundle. Then a connection in the tangent bundle is lifted to the spinor bundle via the Kosmann lift [5, 6, [7]

$$
\Omega=\frac{1}{4} \Lambda^{a b} \gamma_{a} \gamma_{b}
$$

where

$$
\gamma_{a} \gamma_{b}=\eta_{a b}+2 \sigma_{a b}
$$

is the Clifford product in the spinor bundle. The anti-symmetric part of the Clifford product

$$
\sigma_{a b}=\frac{1}{4}\left[\gamma_{a}, \gamma_{b}\right]
$$

carries a representation of the Lorentz group and $\Lambda_{b}^{a}$ is the connection 1-form in the tangent space. The covariant exterior derivative of the Dirac spinor may therefore be written explicitly as

$$
D \psi=d \psi+\frac{1}{2} \Lambda^{[a b]} \sigma_{a b} \psi+\frac{1}{4} Q \psi .
$$

It should be noted that the spin connection $\Omega$ depends on the non-metricity tensor via $q_{a b}$ and the Weyl 1-form $Q$. Thus the Dirac equation in a non-Riemannian spacetime is given by

$$
{ }^{*} \gamma \wedge D \psi+\mu^{*} 1 \psi=0
$$

where the Clifford algebra $\mathcal{C} \ell_{3,1}$-valued 1-form $\gamma=\gamma^{a} e_{a}$ and $\mu=\frac{m c}{\hbar}$.

\subsection{A spinning particle in a non-Riemannian spacetime}

In this section we investigate the motion of a massive, spinning particle in a non-Riemannian spacetime with curvature, torsion and certain components of non-metricity. In order to do that we construct the Dirac Hamiltonian of the particle. This will be done here in the background Schwarzschild metric of a heavy, static body of mass $M$ such as the Sun. In isotropic coordinates $(t, x, y, z)$ the metric is given by円

$$
g=-f^{2} d t^{2}+g^{2}\left(d x^{2}+d y^{2}+d z^{2}\right)
$$

where the metric functions $f$ and $g$ are functions of $r^{2}=x^{2}+y^{2}+z^{2}$ only. The Schwarzschild geometry is specified by the functions

$$
\begin{aligned}
& f=\frac{1+\xi}{1-\xi}, \\
& g=(1-\xi)^{2},
\end{aligned}
$$

\footnotetext{
${ }^{1}$ We use a unit system such that $c=G=1$.
} 
where $\xi=-\frac{M}{2 r}$. The orthonormal co-frame may be cast as

$$
e^{0}=f d t, \quad e^{i}=g d x^{i}, \quad i=1,2,3 .
$$

Then the Levi-Civita connection 1-forms are calculated from (7) as

$$
\omega_{0 i}=-\frac{\left(\partial_{i} f\right)}{f g} e^{0}, \quad \omega_{i j}=\frac{1}{g^{2}}\left[\left(\partial_{j} g\right) e_{i}-\left(\partial_{i} g\right) e_{j}\right] .
$$

Therefore the covariant exterior derivative of the Dirac spinor $\psi$ becomes

$$
\begin{aligned}
D \psi= & e^{0}\left[\frac{\left(\partial_{t} \psi\right)}{f}-\frac{1}{4} Q^{0} \psi-\frac{\left(\partial_{i} f\right)}{f g} \sigma^{0 i} \psi\right] \\
& +e^{i}\left[\frac{\left(\partial_{i} \psi\right)}{g}+\frac{1}{4} Q_{i}+\frac{\left(\partial_{j} g\right)}{g^{2}} \sigma^{i j} \psi\right] \\
& +e^{c} \frac{1}{2} \Omega_{c, a b} \sigma^{a b} \psi
\end{aligned}
$$

where $\Omega_{a b}=K_{a b}+q_{a b}$ and the Weyl 1-form $Q=Q_{a} e^{a}$. After substituting (39) into (33) and using ${ }^{*} e^{f} \wedge e^{a}=-\eta^{f a *} 1$ we obtain

$$
\begin{aligned}
\frac{\gamma^{0}}{f} \frac{\partial}{\partial t}= & \mu \psi+\gamma^{0} \frac{1}{4} Q^{0} \psi \\
& -\gamma^{i}\left[\frac{\left(\partial_{i} \psi\right)}{g}+\frac{1}{4} Q^{i}+\frac{\left(\partial_{j} g\right)}{g^{2}} \sigma^{i j} \psi\right] \\
& +\frac{\left(\partial_{i} f\right)}{f g} \gamma^{0} \sigma^{0 i} \psi-\frac{1}{2} \Omega_{c, a b} \gamma^{c} \sigma^{a b} \psi
\end{aligned}
$$

Now using the identity, with $\gamma_{5}=\gamma^{0} \gamma^{1} \gamma^{2} \gamma^{3}$,

$$
\gamma^{a} \sigma^{b c}=\frac{1}{2} \eta^{a b} \gamma^{c}-\frac{1}{2} \eta^{a c} \gamma^{b}-\frac{1}{2} \epsilon^{a b c d} \gamma_{5} \gamma_{d}
$$

we rearrange this as

$$
\begin{aligned}
\frac{1}{f} \frac{\partial \psi}{\partial t}= & \frac{\gamma^{0} \gamma^{i}}{g}\left[\partial_{i}+\frac{g}{4} Q^{i}+\frac{\partial_{i}(f g)}{2 f g}\right] \psi \\
& +\frac{1}{2} \Omega^{a,}{ }_{a b} \gamma^{0} \gamma^{b} \psi-\mu \gamma^{0} \psi+\frac{1}{4} Q^{0} \psi \\
& -\frac{1}{4} \Omega_{c, a b} \epsilon^{a b c d} \gamma^{0}\left(\gamma_{5} \gamma_{d}\right) \psi
\end{aligned}
$$

Under the definitions $i \gamma^{0}:=-\beta$ and $\gamma^{0} \gamma^{i}:=-\alpha^{i}$, with the constants $\hbar$ and $c$ put in, equation(42) takes the form

$$
\begin{aligned}
i \hbar \frac{\partial \psi}{\partial t}= & -i \hbar \frac{f c}{g} \alpha^{i}\left[\partial_{i}+\frac{g}{4} Q^{i}+\frac{\partial_{i}(f g)}{2 f g}\right] \psi \\
& +\frac{i \hbar f c}{2} \Omega^{a}{ }_{a b} \gamma^{0} \gamma^{b} \psi+f m c^{2} \beta \psi+\frac{i}{4} \hbar c f Q^{0} \psi \\
& -\frac{i \hbar f c}{4} \Omega_{c, a b} \epsilon^{a b c d} \gamma^{0}\left(\gamma_{5} \gamma_{d}\right) \psi .
\end{aligned}
$$


In analogy with the Schrödinger equation

$$
i \hbar \frac{\partial \psi}{\partial t}=H \psi
$$

we write down the Dirac Hamiltonian matrix as

$$
\begin{aligned}
H= & \frac{f}{g} c \vec{\pi} \cdot \vec{\alpha}+f m c^{2} \beta+\frac{i}{4} \hbar c Q^{0}-\frac{i}{4} \hbar f c Q_{i} \alpha^{i} \\
& +\frac{i \hbar f c}{2} K^{a,}{ }_{a b} \gamma^{0} \gamma^{b}+\frac{i \hbar f c}{2} q^{a}{ }_{a b} \gamma^{0} \gamma^{b} \\
& -\frac{i \hbar f c}{4} K_{c, a b} \epsilon^{a b c d} \gamma^{0}\left(\gamma_{5} \gamma_{d}\right)
\end{aligned}
$$

where

$$
\vec{\pi}:=\vec{p}-\frac{i \hbar}{2 f g} \vec{\nabla}(f g)=-i \hbar \vec{\nabla}-\frac{i \hbar}{2 f g} \vec{\nabla}(f g)
$$

The above expression can be further simplified in terms of the irreducible components of non-metricity and torsion. We write

$$
\imath^{a} Q_{a b}=\Lambda_{b}+\frac{1}{4} \imath_{b} Q
$$

so that

$$
q_{a b}^{a,}=\frac{3}{4} Q_{b}-\Lambda_{b}
$$

From (26) and defining

$$
\begin{aligned}
\alpha & =F_{c} e^{c}, \\
\sigma & =\frac{1}{3 !} \sigma_{a b c} e^{a b c}, \\
t_{b} & =\frac{1}{2} t_{a c, b} e^{a c}
\end{aligned}
$$

where $e^{a b}=e^{a} \wedge e^{b}$ and so on, we calculate

$$
\begin{aligned}
K_{a b}^{a,} & =t_{b, a}^{a}+F_{b}, \\
K_{[c, a b]} & =-\frac{1}{6} \sigma_{c a b}=\epsilon_{a b c f} S^{f} .
\end{aligned}
$$

Here $S=S^{a} X_{a}$ is a vector field.

Now when (48), (52) and (53) are substituted in (45) and the identity $\epsilon_{a b c f} \epsilon^{a b c d}=$ $-3 ! \delta_{f}^{d}$ is used, we obtain

$$
\begin{aligned}
H= & \left(\frac{f}{g} c \vec{\pi}-\frac{i}{2} f \hbar c \vec{N}\right) \cdot \vec{\alpha}-\frac{3}{2} i \hbar f c S^{0} \gamma_{5} \\
& +\frac{i}{2} \hbar f c N^{0}+\frac{3}{2} \hbar f c \vec{S} \cdot \vec{\Sigma} \\
& +f m c^{2} \beta
\end{aligned}
$$


where

$$
\begin{aligned}
N_{b} & =t_{b, a}^{a}+F_{b}+\frac{5}{4} Q_{b}-\Lambda_{b}, \\
\Sigma^{i} & =\left(\begin{array}{cc}
\sigma^{i} & 0 \\
0 & \sigma^{i}
\end{array}\right) .
\end{aligned}
$$

As far as we know, only the cases involving axial components of torsion had been studied in the literature. The $N_{a}$ terms in the Dirac Hamiltonian (54) that involve contributions from both the torsion and non-metricity are new.

\subsection{The low energy limit}

If one encounters the situation in which no high momentum states occur, and the momentum of the Dirac particle remains small compared with $m c$ under the influence of all interactions, we have essentially a non-relativistic problem. It therefore becomes valid to look at the problem in the context of 2-component wave functions. The technique for obtaining the Hamiltonian in the low-energy limit is known as the Foldy-Wouthuysen transformation [9]. Far away from the central gravitating body of a mass $M$, it is sufficient to consider a weak limit of the Schwarzschild field (34) with

$$
f \approx 1+\frac{\vec{g} \cdot \vec{r}}{c^{2}}, \quad g \approx 1-\frac{\vec{g} \cdot \vec{r}}{c^{2}}
$$

where

$$
\vec{g}=-G M \frac{\vec{r}}{r^{3}}
$$

Then the Hamiltonian (54) may be written down as

$$
H=m c^{2} \beta+\vartheta+\varepsilon
$$

where

$$
\vartheta=\left(\frac{f}{g} c \vec{\pi}-\frac{i}{2} \hbar f c \vec{N}\right) \cdot \vec{\alpha}-\frac{3 i}{2} \hbar f c S^{0} \gamma_{5}
$$

is the odd operator and

$$
\varepsilon=\frac{i}{2} \hbar f c N^{0}+\frac{3}{2} \hbar f c \vec{S} \cdot \vec{\Sigma}+\vec{g} \cdot \vec{r} m \beta
$$

is the even operator.

For our case here, distinct from [9], in which the odd terms are removed from the Hamiltonian order by order in $1 / m$ and the even operator does not have the mass term, we have the even part $\varepsilon$ necessarily containing a term proportional

to the mass $m$. As a result, the removed terms are the same order in $1 / m$ as the kept terms. This makes the issue of convergence of the approximation scheme 
problematic. It may be recalled that there is another approach to the FoldyWouthuysen transformation (see [10, 11] and references therein). However, it seems that this approach is not applicable to our case because of the difficulty of constructing an anticommuting involution operator (equations $(6),(9)$ in [10] and equation(17) in [11]). Thus in spite of the convergence ambiguity we apply the usual approximation scheme here. Performing the Foldy-Wouthuysen transformation, we obtain the following Hamiltonian to order $1 / m$,

$$
H=\beta\left(m c^{2}+\frac{\vartheta^{2}}{2 m c^{2}}\right)+\varepsilon+\cdots
$$

which gives

$$
\begin{aligned}
H= & \beta\left\{m c^{2}+m \beta \vec{g} \cdot \vec{r}+\frac{f^{2}}{2 m g^{2}}(\vec{\pi})^{2}\right. \\
& -\frac{i \hbar f}{2 m g} \vec{\nabla}\left(\frac{f}{g}\right) \cdot \vec{\pi}+\frac{\hbar f}{2 m g}\left[\vec{\nabla}\left(\frac{f}{g}\right) \times \vec{\pi}\right] \cdot \vec{\Sigma} \\
& +\frac{i f c \hbar}{2} N^{0}-\frac{f^{2} \hbar^{2}}{4 m g} \vec{\nabla} \cdot \vec{N}-\frac{i f^{2} \hbar^{2}}{4 m g}(\vec{\nabla} \times \vec{N}) \cdot \vec{\Sigma}-\frac{i \hbar^{2} f}{4 m g}(\vec{\nabla} f \times \vec{N}) \cdot \vec{\Sigma} \\
& -\frac{i f^{2} \hbar}{2 m g} \vec{N} \cdot \vec{\pi}+\frac{3 i f^{2} \hbar^{2}}{4 m} S^{0} \vec{N} \cdot \vec{\Sigma}-\frac{\hbar^{2} f}{4 m g} \vec{N} \cdot \vec{\nabla} f-\frac{f^{2} \hbar^{2}}{8 m}(\vec{N})^{2} \\
& +\frac{3 f c \hbar}{2} \vec{S} \cdot \vec{\Sigma}-\frac{3 f^{2} \hbar}{2 m g} S^{0} \vec{\pi} \cdot \vec{\Sigma} \\
& \left.+\frac{3 i \hbar^{2} f}{4 m g} \vec{\nabla}\left(f S^{0}\right) \cdot \vec{\Sigma}+\frac{9 f^{2} \hbar^{2}}{8 m}\left(S^{0}\right)^{2}\right\}+\cdots .
\end{aligned}
$$

This Hamiltonian is free of odd operators to order $1 / m$ and consequently to this order its eigenfunctions are 2-component wave functions corresponding to positive and negative energies. For positive energies we have the following Pauli Hamiltonian

$$
H=H_{0}+H_{1}+H_{2}
$$

where

$$
\begin{aligned}
H_{0}= & m c^{2}+m \beta \vec{g} \cdot \vec{r}+\frac{f^{2}}{2 m g^{2}}(\vec{\pi})^{2} \\
& -\frac{i \hbar f}{2 m g} \vec{\nabla}\left(\frac{f}{g}\right) \cdot \vec{\pi}+\frac{\hbar f}{2 m g}\left[\vec{\nabla}\left(\frac{f}{g}\right) \times \vec{\pi}\right] \cdot \vec{\sigma}+\cdots
\end{aligned}
$$

is the (pseudo-)Riemannian part of the Hamiltonian in the low-energy limit,

$$
\begin{aligned}
H_{1}= & \frac{3 f c \hbar}{2} \vec{S} \cdot \vec{\sigma}-\frac{3 f^{2} \hbar}{2 m g} S^{0} \vec{\pi} \cdot \vec{\sigma} \\
& +\frac{3 i \hbar^{2} f}{4 m g} \vec{\nabla}\left(f S^{0}\right) \cdot \vec{\sigma}+\frac{9 f^{2} \hbar^{2}}{8 m}\left(S^{0}\right)^{2}+\cdots
\end{aligned}
$$


contains the axial part of torsion in $H$, and

$$
\begin{aligned}
H_{2}= & \frac{i f c \hbar}{2} N^{0}-\frac{i f^{2} \hbar}{2 m g} \vec{N} \cdot \vec{\pi}+\frac{3 i f^{2} \hbar^{2}}{4 m} S^{0} \vec{N} \cdot \vec{\sigma}-\frac{f^{2} \hbar^{2}}{4 m g} \vec{\nabla} \cdot \vec{N}-\frac{\hbar^{2} f}{4 m g} \vec{N} \cdot \vec{\nabla} f \\
& -\frac{i f^{2} \hbar^{2}}{4 m g}(\vec{\nabla} \times \vec{N}) \cdot \vec{\sigma}-\frac{i \hbar^{2} f}{4 m g}(\vec{\nabla} f \times \vec{N}) \cdot \vec{\sigma}-\frac{f^{2} \hbar^{2}}{8 m}(\vec{N})^{2}+\cdots
\end{aligned}
$$

contains the other components of torsion and certain components of non-metricity and constitutes the original content of this work. Similar calculations have been done in [12 for effects on energy levels of atoms coming from the coupling just the axial part of torsion to the Dirac equation and in [8] for the inertial (acceleration and rotation) and torsional effects in the low energy limit in the context of Einstein-Cartan-Dirac theory in which the torsion tensor is purely axial and in [11, 13] for the test of equivalence principle without torsion.

\section{Discussion}

We have studied the motion of a massive, spinning particle in a background spacetime geometry with torsion and non-metricity. To this end we have written the Dirac equation in such non-Riemannian spacetimes including only certain components of non-metricity. On the other hand all irreducible components of torsion were included for the purpose of comparing our results with those already existing in the literature. After a Foldy-Wouthuysen transformation the Dirac Hamiltonian is the sum of three terms where $H_{0}$ coincides with the original FoldyWouthuysen expression in the limit $f=g=1$ and is independent of both torsion and non-metricity. $H_{1}$ depends only on the axial components of the torsion tensor while the last term $\mathrm{H}_{2}$ is proportional to $N_{a}$ 's that involve both torsion and nonmetricity components. All the contributions to $\mathrm{H}_{2}$ turn out to be of the order of $\hbar$ or of higher orders. Consequently their effect would be too small to be macroscopically significant. However, at the microscopic scales the coupling of a Dirac particle to spacetime non-metricity might have some interesting implications.

\section{Acknowledgement}

This work has been supported in part by TÜBITAK (The Scientific and Technical Research Council of Turkey). We thank Professor R. W. Tucker and Dr. P. Nurowski for discussions. 


\section{References}

[1] T. Dereli, R. W. Tucker, Variational methods and effective actions in string models, Class. Quant. Grav. 4, 791 (1987).

[2] I. M. Benn, T. Dereli, R. W. Tucker, A critical analysis of some fundamental differences in gauge approaches to gravitation, J. Phys. A15, 849 (1982).

[3] F. W. Hehl, J. D. McCrea, E. W. Mielke, Y. Ne'eman, Metric-Affine Gauge Theory of Gravity: Field Equations, Noether Identies, World Spinors, and Breaking of Dilaton Invariance, Phys. Rep. 258, 1 (1995).

[4] T. Dereli, M. Önder, J. Schray, R. W. Tucker, C. Wang, Non-Riemannian gravity and the Einstein-Proca system, Class. Quant. Grav. 13, L103 (1996).

[5] Y. Kosmann, Dérivées de Lie des spineurs, C.R.Acad. Sci. 262 289, 394 (1966).

[6] D. J. Hurley, M. A. Vandyck, On the concepts of Lie and covariant derivatives of spinors, J. Phys. A27, 4569, 5941 (1994), ibid, A28, 1047 (1995).

[7] L. Fatibene, M. Ferraris, M. Francaviglia, M. Godina, A geometric definition of Lie derivative for spinor fields, Proc 6th Int Conf on Diff Geom and Appl, Brno, Czech Republic (1995) (gr-qc/9608003).

[8] P. Singh, L. H. Ryder, Einstein-Cartan-Dirac theory in the low-energy limit, Class. Quan. Grav. 14, 3513 (1997).

[9] L. L. Foldy, S. A. Wouthuysen, On the Dirac Theory of Spin 1/2 Particles and Its Non-Relativistic Limit, Phys. Rev. 78, 29 (1950).

[10] A. G. Nikitin, On exact Foldy-Wouthuysen transformation, J.Phys. A31, 3297 (1998).

[11] Y. Obukhov, Spin, gravity and inertia, Phys. Rev. Lett. 86, 192 (2001).

[12] C. Lämmerzahl, Constraints on space-time torsion from Hughes-Drever experiments, Phys. Letts. A228, 223 (1997).

[13] K. Varjú, L. H. Ryder, The effect of Schwarzschild field on spin 1/2 particles compared to the effect of a uniformly accelerating frame, Phys. Letts. A250 , 263 (1998). 